\title{
Einsatz von im Laserschmelzverfahren hergestellten patientenspezifischen Implantaten in der Mund-, Kiefer- und Gesichtschirurgie
}

\author{
Majeed Rana, Benjamin Singh, Nils-Claudius Gellrich
}

\section{Zusammenfassung}

Heutzutage stehen modernste Techniken zur Verfügung, durch die mithilfe einer digitalen präoperativen Planung patientenspezifische Implantate (PSI) hergestellt werden können. Vorrangig ist hier das selektive Laserschmelzverfahren auf Titanpulverbasis zu nennen, das sowohl zur Herstellung patientenspezifischer Implantate beliebiger Geometrie und innerer Struktur für Orbita- und Mittelgesichtsrekonstruktionen als auch für jede andere anatomische Region geeignet ist. Alternativ können virtuelle Planungen auch durch die CNC-Frästechnik (CNC: computerized numerical control), Spritz- oder Formentechniken in Implantate umgesetzt werden. Diese Innovationen führen zur Herstellung einer perfekt formstabilen patientenspezifischen Implantatgeometrie. Darüber hinaus kann das Einsetzen und die intraoperative Positionierung dieser Implantate mithilfe dieser Technik gesteuert werden, durch die der gesamte Kreislauf der präoperativen Defektanalyse, patientenspezifischen Implantatherstellung und Insertion von Implantaten qualitätsgesichert werden kann.
Patient-Specific Implants Prepared in the Laser Fusion Procedure: Use in Oral, Maxillary and Facial Surgery

Highly modern techniques are available for computer-supported preoperative digital planning of the direct fabrication of patient-specific implants (PSI). The laser fusion procedure is suitable for producing patient-specific implants of any geometry and internal structure for orbital and midface reconstructions, but also for any other anatomical region, all based on titanium powder. These digital plans can control CNC milling (CNC: computerized numerical control) cutters or lead to implants produced by spraying or moulding techniques. This innovation leads to the production of perfectly formed and stable patient-specific orbital implants of the required geometry. The insertion and intraoperative positioning of these implants can be controlled using this technique to ensure the quality of the entire process: preoperative defect analysis, patientspecific production of implants, and insertion of implants.

\section{Einleitung}

In der Versorgung von Gesichtsschädeldefekten infolge von Verletzungen durch Gewalteinwirkung (Trauma), bösartigen Geschwülsten (Tumoren) sowie erworbenen oder angeborenen Fehlbildungen erfolgt die primäre Rekonstruktion mit dem Ziel einer Wiederherstellung des Gesichtsschädels und der bedeckenden

OP-JOURNAL 2016; 32: 241-246

(c) Georg Thieme Verlag KG Stuttgart · New York DOI http://dx.doi.org/10.1055/s-0042-123202

nen und somit die Notwendigkeit einer späteren Korrektur des Operationsergebnisses im Rahmen eines Folgeeingriffs minimiert wird $[9,18,22]$. So sind die Eingriffe weniger invasiv, die Operationszeiten können z.T. erheblich verkürzt und die Gesichtsschädeldefekte besser rekonstruiert werden. Durch den Einsatz der patientenspezifischen Implantate (PSI) ist es nun möglich, defektorientiert und individuell zu versorgen. Die Eingriffe werden dadurch präziser, es werden geringere Mengen an alloplastischen Materialien verwendet, die Zugänge zum Einbringen dieser Implantate werden kleiner und schonender für das umliegende Gewebe (minimalinvasiv). Die Aufenthaltszeiten können verkürzt und bessere klinische Ergebnisse erreicht werden.

\section{Traumatologie}

Die originalgetreue Rekonstruktion des Gesichts durch eine virtuelle Planung wird bei unilateralen Frakturen inzwischen routinemäßig durch eine Spiegelung der nicht betroffenen Seite erreicht, sodass im Vorfeld der Operation patientenspezifische Implantate hergestellt werden können [7]. Bei sekundären Rekonstruktionen, die sich aufgrund der Weichteilatrophien und Vernarbungen schwieriger gestalten, wird der gleiche Ansatz gewählt [18]. Hierbei werden in der präoperativen Planung zusätzlich die dislozierten Knochen betrachtet und ideale Linien für die Osteotomie geplant. Schwieriger gestaltet sich die Rekonstruktion von bilateralen und mittellinienüberschreitenden Traumata wie komplexen Mittelgesichtsfrakturen oder panfazialen Frakturen, da hier keine Referenz für die Planung des Rekonstruktionsergebnisses im Sinne unbetroffener Strukturen vorliegt. Aber auch für diesen Bereich werden inzwischen Algorithmen entwickelt, die ein auf Normschädeln basierendes Modell der rigiden und elastischen Deformation verwenden, um eine virtuelle Rekonstruktion zu ermöglichen. 


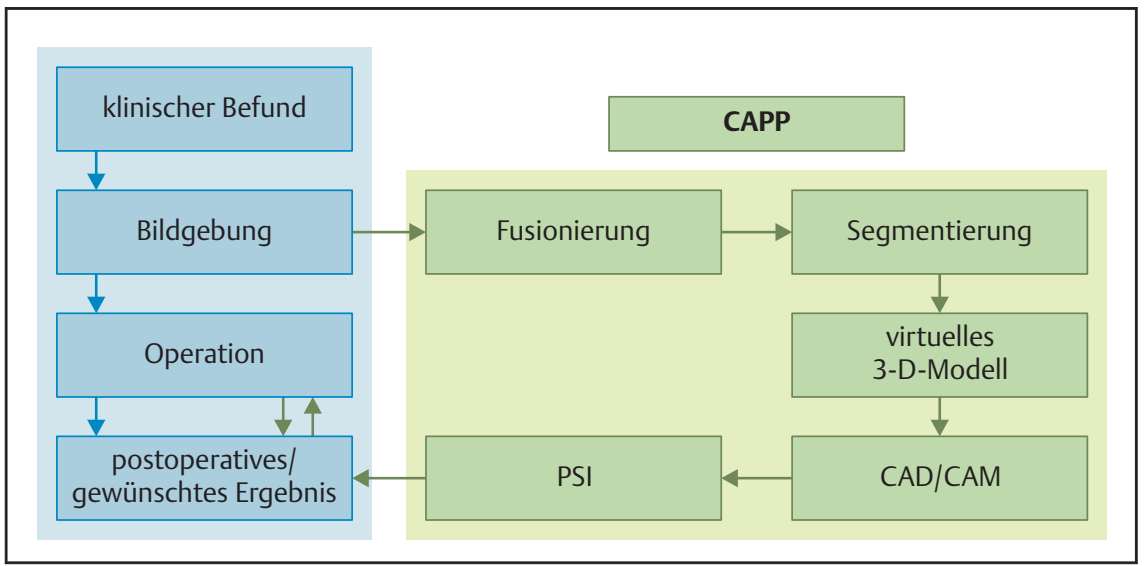

Abb. 1 Ablauf der patientenspezifischen Planung in der computerassistierten Chirurgie.

Weitere Vorteile, welche die CAS in der Traumatologie mit sich bringt, liegen darin, dass aufgrund der Überprüfbarkeit des Erfolgs der Rekonstruktion die Operationszeit durch vorgefertigte, passgenaue patientenspezifische Implantate verkürzt werden kann. Hinzu kommt, dass durch den Einsatz der PSI mithilfe von intraoperativer Navigation eine höhere Präzision erreicht werden kann $[5,23]$.

\section{Kraniosynostosen}

Verknöchert eine Naht oder mehrere Nähte des Schädeldachs (Kalotte) im frühen Lebensabschnitt, ist ein Eingriff und Eröffnung der Kalotte notwendig, um das normale Wachstum des Gehirns zu ermöglichen. Dabei werden Teile der Schädelkalotte sowie die frühzeitig verknöcherte Naht entfernt, um das physiologisch vorgesehene Volumen des Schädels zu ermöglichen. Der anschließende Schritt sieht eine Rekonstruktion des Schädels in der gewünschten Form vor. Dabei werden die gelösten und gelockerten Teile der Kalotte im bisherigen Verfahren händisch und nach Empfinden des jeweiligen Operateurs zu der gewünschten Schädelform modelliert und osteosynthetisch verplattet oder verdrahtet.

Der Einzug der CAD-/CAM-Technik (computer-aided design/manufacturing) ermöglicht eine in diesem Zusammenhang stehende, digitale Bewertung der Anomalie sowie das fehlende und zu erreichende Volumen des Schädels [15,24, 26]. Aufgrund dieser patientenspezifischen digitalen Analysen wird im 1. Schritt eine Schablone (CuttingGuide) mittels verschiedener CAD-/CAM-Techniken geplant $[1,16]$. Während der Operation gibt dieser CuttingGuide die Schnittvektoren der Osteotomie und zu entfernenden Teile der Kalotte vor, indem er als eine Art Helm auf den Schädel des Patienten gesetzt wird. Da dieser Leitfaden im Vorfeld des Eingriffs anhand der bildgebenden Datensätze des Patienten geplant wird, ist eine exakte Passform ebenso sichergestellt wie die optimalen Schnittvektoren für diesen einzelnen Patienten.

Nach der Eröffnung der Kalotte und der Kontrolle des gewünschten Volumens wird die Rekonstruktion ebenfalls mittels patientenspezifischer Implantate durchgeführt. Im Vorfeld der Operation wird hierzu digital ein Gerüst entworfen, welches die Form des operierten Schädels vorgibt und sich an der Form der normalen physiologischen Situation orientiert. Dieses Gerüst besteht aus einer milchsäurebasierten Gitterstruktur, die als Fixierungsreferenz für die gelösten Knochensegmente dient und somit die Einheilposition und Wachstumsrichtung vorgibt (Abb. 2).

Die Vorteile dieser patientenspezifischen Planung und Versorgung liegen in der Verringerung von individuellen Fehlerquellen durch eine vorgegebene, individuelle Planung der Operationsschritte [14]. Nicht zuletzt bietet das in dem patientenspezifischen Implantat gespeicherte Wunschvolumen des Schädels eine hohe Erfolgschance für eine normale Entwicklung des Patienten [3, $8,11]$.

\section{Dentale Implantate}

Der Einsatz von patientenspezifischen Implantaten in der dentalen Implantologie findet hauptsächlich in der prothetisch orientierten Rehabilitation der Kaufunktion sowie der Erhaltung und Schonung vitaler Strukturen Anwen- dung. Darüber hinaus steht die Verringerung postoperativer Komplikationen sowie die Verbesserung funktioneller und ästhetischer Ergebnisse im Vordergrund der dentalen PSIs. Der Einsatz der digitalen Planung im Vorfeld wirkt einer fehlerhaften Implantatpositionierung sowie der Verletzung empfindlicher Strukturen entgegen, bei unterschiedlichsten Behandlungsindikationen $[13,14]$. Der Erfolg bisheriger Methoden, wie die Insertion von Zygomaimplantaten nach einer ablativen Tumorchirurgie im Oberkiefer oder auch die Implantatinsertion von konventionellen Dentalimplantaten nach mikrovaskulärer Knochentransplantation sind stark vom Können des Operateurs abhängig. Die Insertion des dentalen PSIs ist bei einer sorgfältigen präoperativen Planung deutlich angenehmer als die Insertion von Zygomaimplantaten

Den Erfolg einer implantatprothetischen Rehabilitation bestimmt die exakte Planung der Implantatposition und im Allgemeinen die prothetischen Versorgungsmöglichkeiten. Die Planung beginnt hier mit dem Endresultat, auf dessen Informationen alle Schritte bis zu diesem Endergebnis basieren und geplant werden. Dieses sog. „Backwards Planning" wird abgerundet durch den Einsatz digitaler Planungssoftware vor dem Eingriff und der abschließenden Dokumentation anhand aller einzelnen Planungsschritte (Abb. 3).

\section{Tumorchirurgie}

In der ablativen Tumorchirurgie werden im Zuge der präoperativen Planung zunächst die multimodalen Bilddatensätze fusioniert, um einerseits die knöcherne Beteiligung, andererseits die Tumorausdehnung bestmöglich zu bestimmen. Diese sog. Segmentierung bildet die Grundlage zur Bestimmung der Resektionsgrenzen inklusive des Sicherheitsabstands. Im gleichen Zuge wird anhand der Segmentierung und des Bildmaterials die virtuelle Rekonstruktion geplant und simuliert. Diese ist nicht nur notwendig, um die patientenspezifischen Implantate herzustellen, sondern spielt auch während der intraoperativ navigierten Insertion von PSI eine wichtige Rolle. Hierbei erleichtern zuvor segmentierte anatomische Strukturen, wie bspw. die A. carotis interna oder die Sehnerven, die Navigation erheblich und erhöhen zudem die Sicherheit des Chirurgen [20]. Gleichzeitig hat dieser die Möglichkeit, intraoperative Informationen 


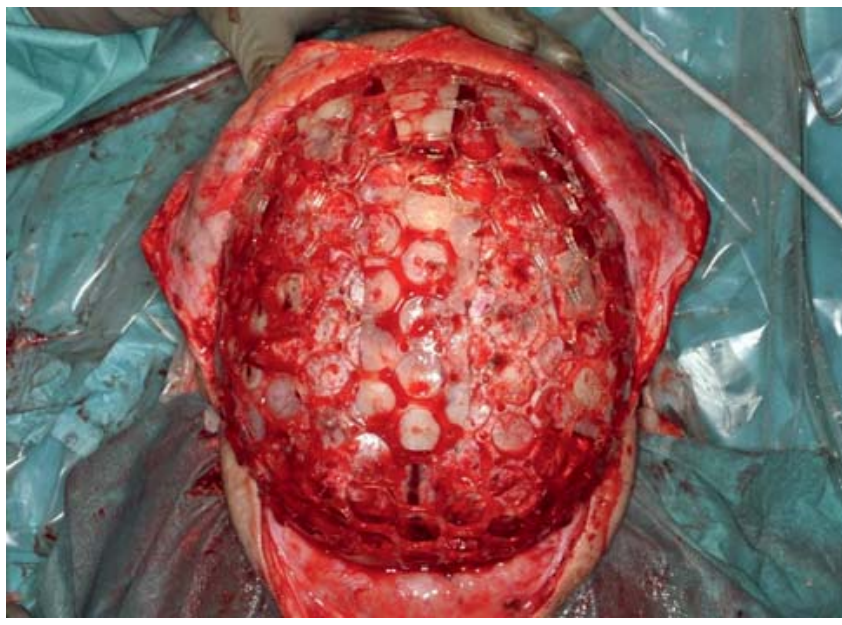

Abb. 2 Milchsäurebasiertes Gittergerüst zur Fixierung der Knochensegmente.

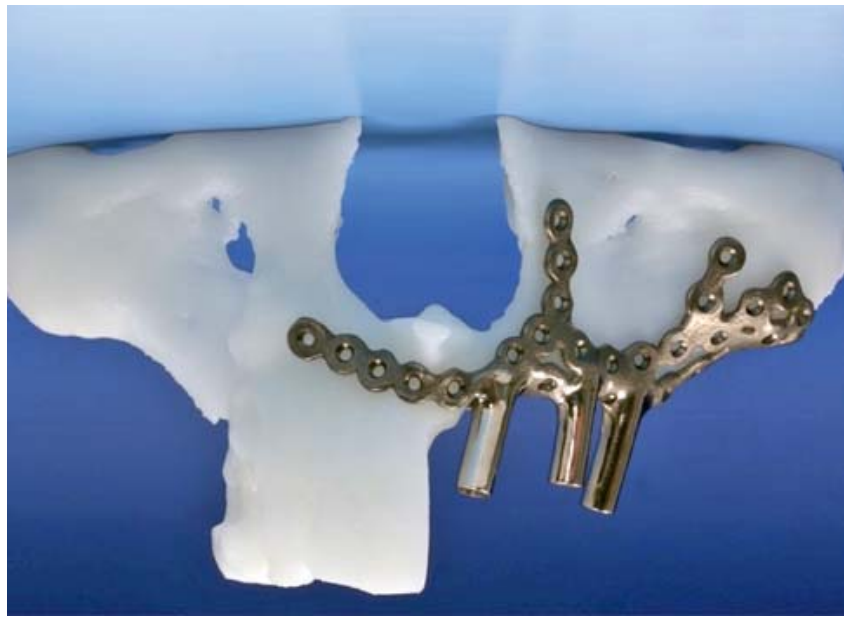

Abb. 3 Durch das „Backwards Planning“ hergestelltes maxilläres, patientenspezifisches Dentalimplantat. direkt auf dem Navigationsmodul während der Operation zu markieren und zu speichern. Diese Informationen sind vor allem für den Strahlentherapeuten von Bedeutung, sodass dieser die markierten, intraoperativ aus anatomischen Gründen nicht resektablen Tumoranteile oder den nicht einhaltbaren Sicherheitsabstand in seiner Bestrahlungsplanung berücksichtigen kann [9]. Ohne die intraoperative Navigation ist die Benennung und Zuordnung der Randschnitte (Tumor Mapping) erschwert, wodurch die entnommene Probe u.U. nicht mehr anatomisch korrekt lokalisiert werden kann. Hier bietet die CAS den Vorteil, dass die Randschnitte virtuell markiert und benannt werden können. Dadurch wird auch die Zuordnung der histologischen Ergebnisse deutlich vereinfacht. Sollte im Langzeitverlauf ein Re-Staging notwendig sein, können die vormals gespeicherten Daten verwendet und mit den neu erhobenen Datensätzen fusioniert und verglichen werden [21].

Nach erfolgreicher Resektion ist es oft notwendig, eine funktionelle und ästhetische Versorgung des Resktionsgebiets durchzuführen. Da diese Strukturen oft funktionelle Beeinträchtigungen nach sich ziehen, ist hier eine optimale wiederherstellende Versorgung notwendig. Diese wird, wie bereits erwähnt, zunächst patientenspezifisch virtuell mittels patientenspezifischer Implantate geplant und anschließend mithilfe der Navigation in die richtige Position gebracht und fixiert. Durch die individuelle Planung jedes Patienten kann die jeweils beste funktionelle und ästhetische Lösung gewährleistet werden. Besonders in einem Bereich der Chirurgie, die weder standardisierten Abläufen noch Me- thoden unterliegt, sind individuelle Lösungen von entscheidender Bedeutung für den klinischen und ästhetischen Rehabilitationserfolg.

\section{Unterkieferrekonstruktion}

Die Unterkieferrekonstruktion kommt besonders in der Tumorchirurgie häufig zum Einsatz. Verliert ein Patient durch die Entfernung eines Tumors wesentliche Anteile an Zähnen und zahntragendem Knochen des Unterkiefers, ist eine Wiederherstellung der Funktion und Ästhetik notwendig [4]. Hierbei bewährte sich für die Rekonstruktion das mikrovaskuläre Fibulatransplantat. Die entfernten Knochen werden mit neuem patienteneigenem Knochen ersetzt. Dort können im weiteren Werdegang dentale Implantate eingesetzt werden, sodass eine adäquate Rehabilitation gewährleistet wird [17].

Die Position des neuen Knochens wird dabei über eine computerassistierte Rückwärtsplanung bestimmt. Am Computer werden in einem neuen, eigens entwickelten Verfahren die Dimensionen dentaler Implantate virtuell in Beziehung zu den bestehenden Zähnen gesetzt, um später eine optimale Zahnverschlüsselung zu sichern.

Im Rahmen der virtuellen Simulation kann im Laufe der präoperativen Planung schon berücksichtigt werden, an welcher Stelle das Knochentransplantat später positioniert werden soll, sodass dieses im Laufe des operativen Eingriffs mühelos eingesetzt werden kann [19]. In diesem Zusammenhang werden patientenspezifische defektorientierte physikalische Schablonen (Templates) ein- gesetzt, die während der Entnahme des Transplantats direkt auf den Knochen aufgelegt werden können, um so das Transplantat passgenau $\mathrm{zu}$ trimmen. Eine weitere Schablone, in manchen Fällen sogar dieselbe Schablone, kann dann anschließend verwendet werden, um das Transplantat in seine neue Position einzusetzen, da die Lageposition des Knochens genau abgeschätzt werden kann (Abb. 4).

\section{Primäre Orbitarekonstruktion}

Ist aufgrund einer Verletzung durch Gewalteinwirkung oder durch die Entfernung eines Tumors eine Orbitarekonstruktion notwendig, liegt das Ziel in der originalgetreuen Rekonstruktion der Orbita in Form und Volumen. Hierbei soll sowohl die funktionelle als auch die ästhetische Wiederherstellung erreicht werden $[7,25]$.

Durch die Reduktion des Orbitavolumens kann im Anschluss an eine Tumorresektion und eine Primärrekonstruktion der Weichteildefekt idealerweise behoben werden. Die Folgen eines Weichteildefekts sind u.a. die Wahrnehmung von Doppelbildern, eine Erhöhung des Augeninnendrucks oder auch neurologische Störungen. Neben den funktionellen Beeinträchtigungen kann zusätzlich die Ästhetik durch eine Gesichtsasymmtrie, einem En- oder Exophthalmus oder einem Bulbushoch- oder -tiefstand beeinträchtigt werden.

Aufgrund der komplexen anatomischen Strukturen stellt eine Orbitarekonstruktion den Chirurgen vor eine große Herausforderung. Eine 3-dimensionale $\mathrm{Vi}$ sualisierung der Strukturen und eine 


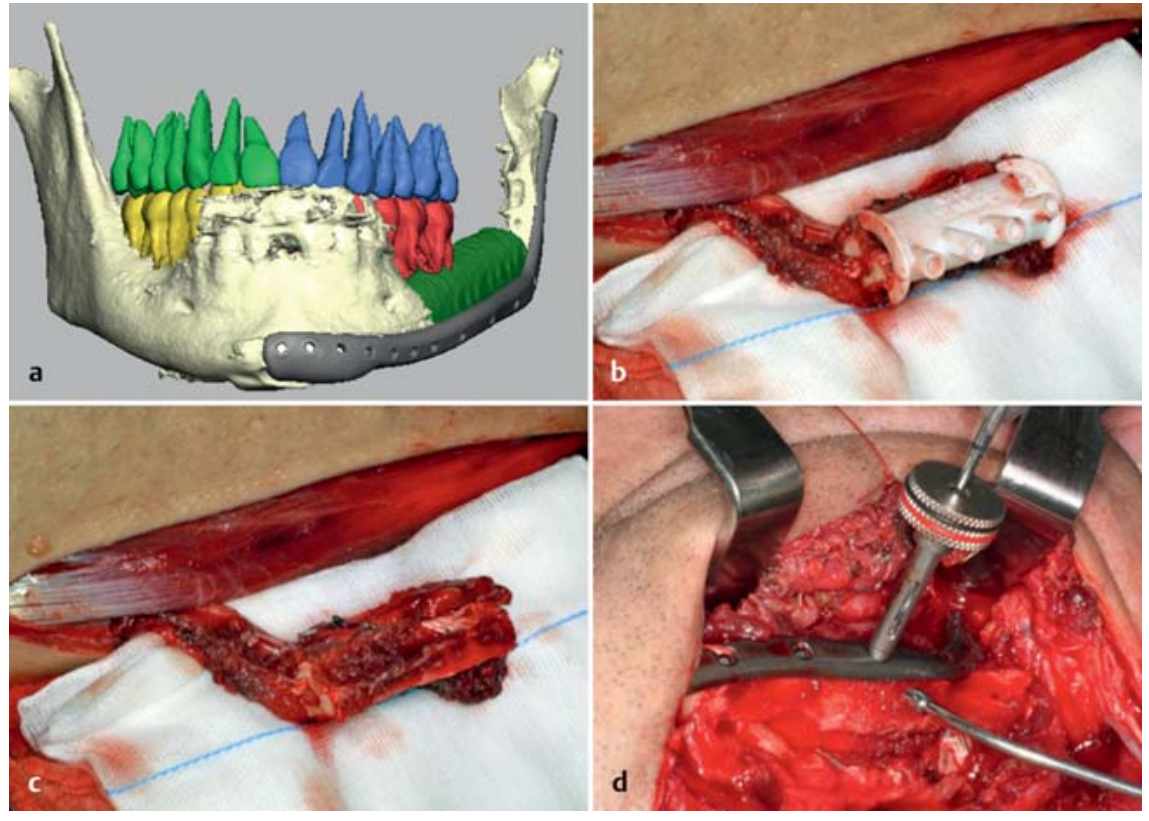

Abb. $\mathbf{4}$ a bis $\mathbf{d}$ Schritte von der digitalen Planung über Schablonen bis zum Implantat.

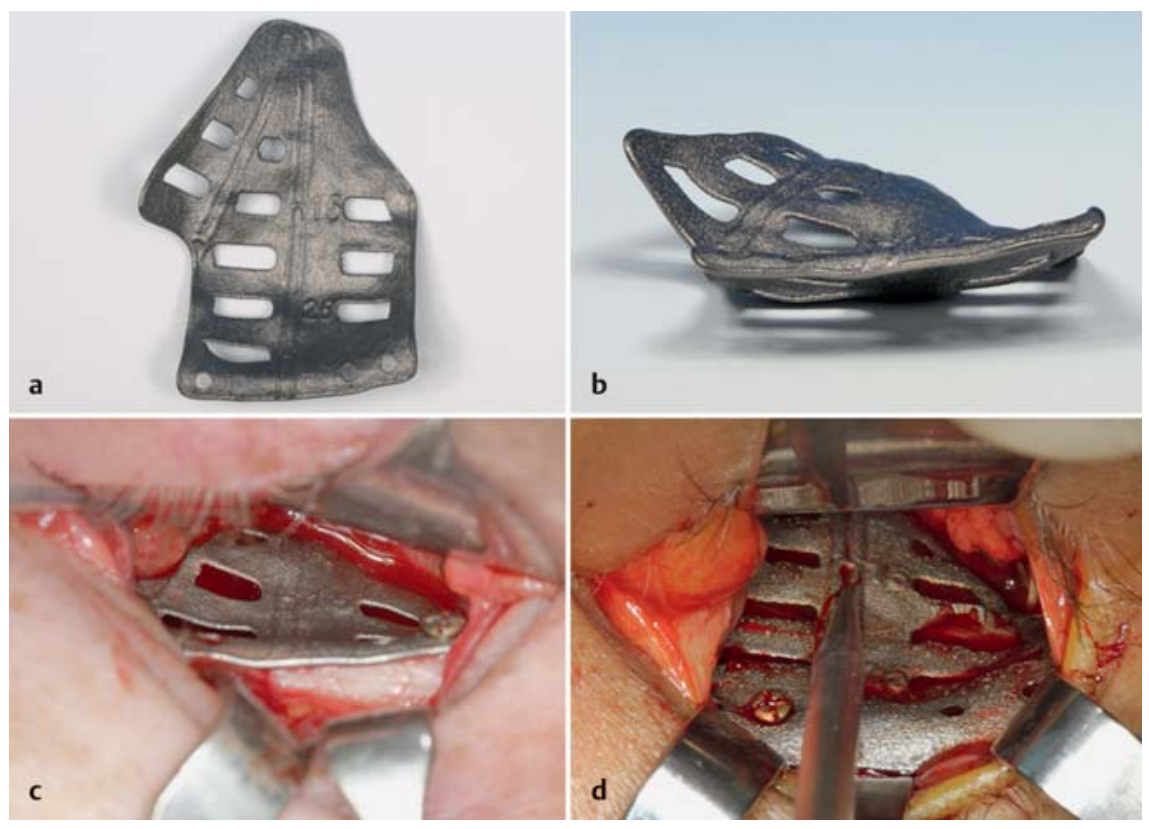

Abb. $\mathbf{5}$ a bis d Lagekontrolle des Implantats mithilfe einer Navigation.

sorgfältige präoperative Planung ermöglichen einen sicheren chirurgischen Eingriff und erhöhen die Erfolgsaussichten immens. Hierbei kommt die Spiegelung der nicht betroffenen kontralateralen Gesichtshälfte zum Einsatz [18]. Der Originaldatensatz wird im 1 . Schritt seitenverkehrt dupliziert und anschließend wird das Duplikat mit dem Original fusioniert. Zusätzlich zur Planung und Entwicklung eines patientenspezifischen Implantats dient der Datensatz während der intraoperativen Navigation als eine Art virtuelle Schablone. Dadurch kann mithilfe eines Pointers sichergestellt werden, ob das Implantat anatomisch korrekt eingesetzt worden ist (Abb. 5).

\section{Sekundäre Orbitarekonstruktion}

Die Folge einer Orbitaoperation kann eine dramatische Veränderung der Gesichtsgeometrie beinhalten. Dadurch ist ein sekundärer Korrektureingriff für die Wiederherstellung der Funktion und Ästhetik oftmals notwendig. Besonders anophthalmische Patienten, die mit einer Orbitaprothese versorgt sind und deren
Lidfunktion erhalten ist, haben ästhetische Einbußen (Einsinken des Augapfels oder der Orbitaprothese in die Augenhöhle).

Neben den chirurgischen Fähigkeiten spielen die Form des Orbitaimplantats, die adäquate Position des Implantats wie auch ein ausreichendes Weichgewebslager eine bedeutende Rolle.

Kleinere Defekte lassen sich i.d.R. ohne größere Komplikationen beheben. Im Gegensatz dazu können große Defekte nach einer ablativen Tumorchirurgie Weichteildeformationen und Gesichtsasymmetrien verursachen [10].

Hierbei bietet der Einsatz von präoperativer Planung und CAS einen sinnvollen Lösungsansatz. Mithilfe der Planung von PSI findet eine sog. Rückwärtsplanung statt. Das PSI wird so designt, dass zuerst das ästhetisch wünschenswerte Endergebnis simuliert wird, indem das Ausmaß des Weichteilgewebes dargestellt wird. Ausgehend von diesem Ziel wird rückwärts geplant, inwieweit die knöchernen Strukturen rekonstruiert werden müssen, um das orbitale Volumen $\mathrm{zu}$ reduzieren und so das Ziel zu erreichen. Diese Form der Planung stellt einen deutlich erhöhten Aufwand dar, aufgrund der Komplexität, da die knöcherne Struktur der Orbita und das periorbitale Weichgewebe berücksichtigt werden müssen. Hierunter fallen die extraokuläre Muskulatur, das den Kochen umgebende Fett- und Weichgewebe sowie das durch die Operation entstandene minderwertige Narben- und Bindegewebe.

Trotz hochauflösender CT- und MRTAufnahmen kann nicht jede Weichgewebsstruktur und deren elastisches Verhalten berücksichtigt werden. Jedoch lassen sich diese Probleme mithilfe der Planung und einer gewissen Überkorrektur kompensieren. Durch den Einsatz der Finite-Elemente-Methodik können die besagten Strukturen so miteinander verknüpft werden, dass das komplexe $\mathrm{Zu}$ sammenwirken der Strukturen annähernd simuliert werden kann und somit die dynamischen Veränderungen durch die einzelnen Arbeitsschritte ersichtlich werden. Im Einzelnen bedeutet dies, dass durch eine manuelle Verschiebung der virtuellen Orbitaprothese in die zu erwartende Position der Algorithmus automatisch die Form und Lage der knöchernen Begrenzungen berechnet. Diese Abweichungen der knöchernen Begren- 
zungen werden durch das Verfahren des Rapid Prototyping ausgeglichen, indem ein PSI-Konstrukt fabriziert wird.

Im Anschluss an die Operation erfolgt eine Evaluation der Ergebnisse anhand der Fusionierung von präoperativen und postoperativen CT-Datensätzen. Zusätzlich ist ein Algorithmus notwendig, der das intraorbitale Volumen misst, sodass im Nachgang sichergestellt werden kann, ob tatsächlich eine Volumenreduktion erfolgt ist.

\section{Dysgnathien - orthognathe Chirurgie}

Die orthognathe Chirurgie ist ein wesentliches Einsatzgebiet der computerassistierten Planung (CAPP) und CAS, wobei die CAPP von Dysgnathien der konventionellen Planung überlegen sein kann $[27,28]$. In der Behandlung spielt neben der funktionellen Verbesserung der mit der Dysgnathie einhergehenden Störungen, wie der Kaufunktion, auch die Ästhetik eine Rolle.

Eine Umstellungsosteotomie kann mithilfe der virtuellen Planung detailliert geplant und die Verschiebungen der Kiefersegmente virtuell simuliert werden, bis eine zufriedenstellende Therapiestrategie vorliegt. Gerade bei anatomisch schwer zu erreichenden Regionen, wie der Le-Fort-III-Ebene, können die Verlagerungsstrecken und Positionierungen der Knochenabschnitte präoperativ geplant werden.

Nach Erstellung der 3-dimensionalen Bilddaten mittels CT oder digitaler Volumentomografie (DVT) wird z.B. ein Autosegmentierungsalgorithmus eingesetzt, um die virtuelle Le-Fort-I-Osteotomie des Oberkiefers und die bisagittale Spaltosteotomie, z.B. nach Obwegeser, vorzubereiten. Im nächsten Schritt werden Gipsmodelle des Zahnbogens vom Ober- und Unterkiefer des Patienten durch einen 3-dimensionalen dentalen Oberflächenscanner gescannt und in die initialen CT- oder DVT-Datensätze importiert, sodass die bildliche Darstellung der Zähne verbessert wird. Dieser Schritt ist entscheidend für die Okklusionskontrolle.

$\mathrm{Zu}$ Beginn der virtuellen Planung muss die gewünschte Position des Oberkiefers festgelegt werden. Anschließend wird ein PSI geplant, welches die neue Position des Oberkiefers in Bezug auf die Schädelbasis sichert. Dabei wird ein Implantat für das Mittelgesicht geplant. Die
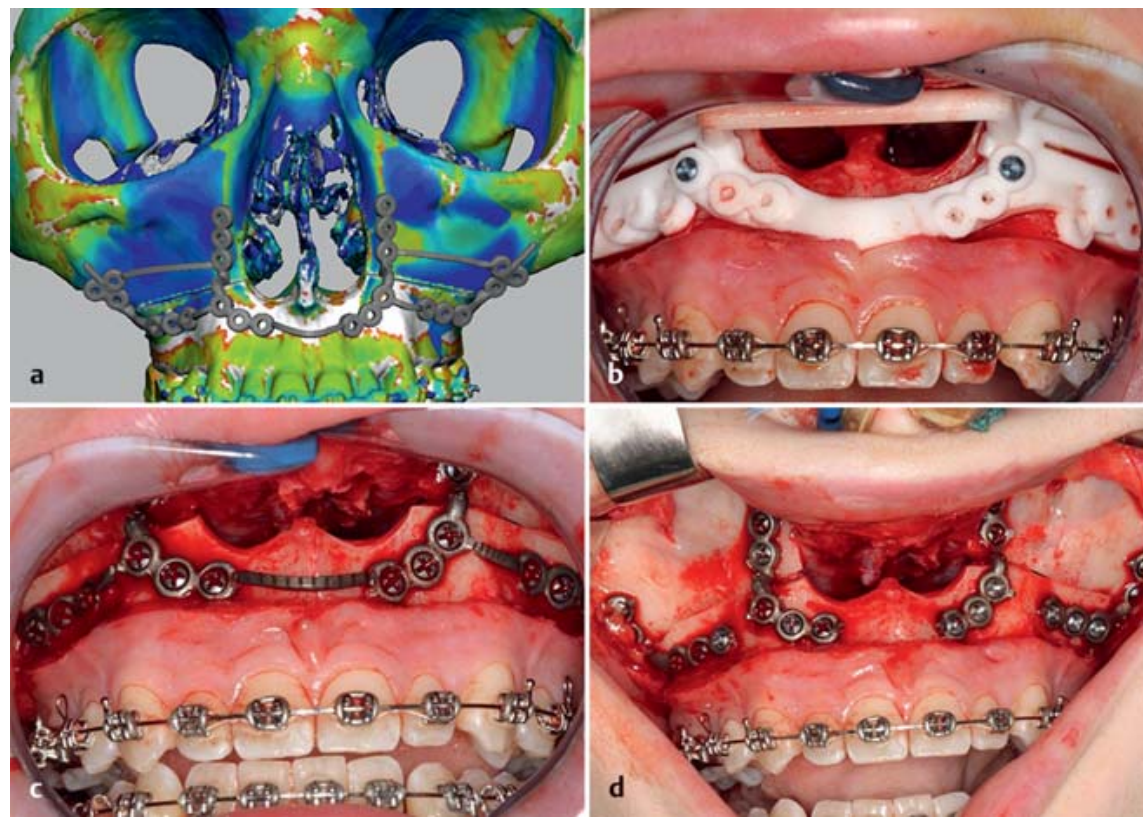

Abb. $\mathbf{6 a}$ bis $\mathbf{d}$ a Ermittlung der Knochendicke und optimalen Schraubenposition mittels einer Heatmap, b Anlegen der Schnitt- und Bohrschablone, c Fixierung des patientenspezifischen Implantats, $\mathbf{d}$ endgültige Form und Lage des PSIs.

Schnitt- und Bohrschablonen geben die Osteotomielinien und die zukünftige Schraubenposition vor. Für den Endsplint wird eine Boole-Verknüpfung durchgeführt, um aus dem vorläufigen virtuellen Splint einen tatsächlichen Splint zu erhalten, um diesen dann mithilfe eines 3-D-Druckers auszudrucken (Abb.6). Des Weiteren kann ein photorealistischer 3-D-Oberflächenscanner genutzt werden, um das Weichgewebe des Gesichts des Patienten darzustellen und mit den präoperativen anatomischen Daten zu fusionieren, wodurch die erwarteten Ergebnisse besser visualisiert werden können. Während der Operation wird die präoperativ geplante Position des Oberkiefers nur mithilfe des PSIs befestigt.

\section{Ausblick}

Durch die rasanten technischen Entwicklungen ist es inzwischen möglich, genauere, kostengünstigere und benutzerfreundlichere Systeme zu entwickeln, die weder lange Einarbeitungszeiten benötigen, da die Anwendung intuitiv nachvollziehbar gestaltet wurde, noch längere Behandlungszeiten nach sich ziehen, da die Planung schnell umzusetzen ist.

Zudem können durch diese technischen Entwicklungen auch bisherige Schwächen der Systeme beseitigt werden, wie bspw. Plattenfrakturen oder eine nicht ideale Passform, da es inzwischen auch
Verfahren gibt, die eine höchstmögliche Stabilität und Passgenauigkeit der PSI möglich machen. Somit können heute schon intelligente Implantate entwickelt werden, die optimal an die Bedürfnisse des Patienten und die anatomischen Gegebenheiten angepasst sind. Hier ist in erster Linie das Electron Beam Melting (EBM) zu nennen. Bisher wird dieses Verfahren vor allem für die Rekonstruktion des Hirnschädels eingesetzt. Aufgrund der bisherigen Erfolge mit dieser Technik soll sie nun auch in der kraniofazialen Chirurgie eingesetzt werden.

\section{Interessenkonflikt: Nein}

\section{Literatur}

${ }^{1}$ Burge J, Saber NR, Looi T et al. Application of CAD/CAM prefabricated age-matched templates in cranio-orbital remodeling in craniosynostosis. J Craniofac Surg 2011; 22: 1810 1813

2 Carl AL, Khanuja HS, Sachs BL et al. In vitro simulation. Early results of stereotaxy for pedicle screw placement. Spine (Phila Pa 1976) 1997; 22: 1160-1164

3 Chim H, Wetjen N, Mardini S. Virtual surgical planning in craniofacial surgery. Semin Plast Surg 2014; 28: 150-158

${ }^{4}$ Eckardt A, Swennen GR. Virtual planning of composite mandibular reconstruction with free fibula bone graft. J Craniofac Surg 2005; 16: 1137-1140

${ }^{5}$ Essig H, Dressel L, Rana $M$ et al. Precision of posttraumatic primary orbital reconstruction using individually bent titanium mesh with and without navigation: a retrospective study. Head Face Med 2013; 9: 18

${ }^{6}$ Gellrich NC, Schramm A, Hammer B et al. Computer-assisted secondary reconstruction of 
unilateral posttraumatic orbital deformity. Plast Reconstr Surg 2002; 110: 1417-1429

${ }^{7}$ Hanasono MM, Jacob RF, Bidaut L et al. Midfacial reconstruction using virtual planning, rapid prototype modeling, and stereotactic navigation. Plast Reconstr Surg 2010; 126 2002-2006

${ }^{8}$ Khechoyan DY, Saber NR, Burge J et al. Surgical outcomes in craniosynostosis reconstruction: the use of prefabricated templates in cranial vault remodelling. J Plast Reconstr Aesthet Surg 2014; 67: 9-16

${ }^{9}$ Kokemüller H, See C, Essig H et al. Rekonstruktion komplexer Mittelgesichtsdefekte durch individualisierte Titanimplantate. HNO 2011; 59: 319-326

10 Lauer G, Pradel W, Schneider M et al. Sekundäre Mittelgesichtsrekonstruktion mittels verschiedener Operationsverfahren unter Nutzung der computergestützten Chirurgie. Mund Kiefer GesichtsChir 2006; 10: 325-329

${ }^{11}$ Mardini S, Alsubaie S, Cayci C et al. Three-dimensional preoperative virtual planning and template use for surgical correction of craniosynostosis. J Plast Reconstr Aesthet Surg 2014; 67: 336-343

12 Merloz P, Tonetti J, Pittet L et al. Computer-assisted spine surgery. Comput Aided Surg 1998; 3: 297-305

${ }^{13}$ Miller RJ, Bier J. Surgical navigation in oral implantology. Implant Dent 2006; 15: 41-47

${ }^{14}$ Miller RJ. Navigated surgery in oral implantology: a case study. Int J Med Robot 2007; 3: 229-234

15 Mommaerts MY, Jans G, Sloten JV et al. On the assets of CAD planning for craniosynostosis surgery. J Craniofac Surg 2001; 12: 547-554

16 Pappa H, Richardson D, Webb AA et al. Individualized template-guided remodeling of the fronto-orbital bandeau in craniosynostosis corrective surgery. J Craniofac Surg 2009; 20: $178-179$

${ }^{17}$ Rana M, Warraich R, Kokemüller $H$ et al. Reconstruction of mandibular defects - clinical retrospective research over a 10-year period. Head Neck Oncol 2011; 3: 23

18 Schmelzeisen R, Schramm A. Computer-assisted reconstruction of the facial skeleton. Arch Facial Plast Surg 2003; 5: 437

19 Schramm A, Gellrich NC. Intraoperative Navigation und computerassistierte Chirurgie. In: Schwenzer N, Hrsg. Mund-Kiefer-Gesichtschirurgie. Stuttgart: Thieme; 2011

${ }^{20}$ Schramm A, Gellrich NC, Naumann S et al. Non-invasive referencing in computer-assisted surgery. MBEC 1999; 37: 644-645

${ }^{21}$ Schramm A, Gellrich NC, Schön R et al. Advantages of computer-assited Surgery in the Treatment of cranio-maxillofacial Tumors. In: Lemke $\mathrm{HU}$, Vannier MW, Inamura K, Farman AG, eds. CARS'99 - Proceedings of the 13th Int. Congress and Exhibition, Paris 23.-26. 6. 1999. Amsterdam: Elsevier; 1999: 903-907

22 Schramm A, Gellrich NC, Schmelzeisen R. Navigational surgery of the facial skeleton. Berlin, Heidelberg, New York: Springer; 2006

23 Schramm A, Wilde F. Die computergestützte Gesichtsschädelrekonstruktion. HNO 2011; 59: 800-806

${ }^{24}$ Seruya M, Borsuk DE, Khalifian S et al. Computer-aided design and manufacturing in craniosynostosis surgery. J Craniofac Surg 2013; 24: 1100-1105

25 Terzic A, Scolozzi P. Image guided surgical navigation integrating "mirroring" computational planning based on intra-operative cone-beam CT imaging: a promising new ap- proach for management of primary bilateral midfacial fractures. Comput Aided Surg 2011; 16: 170-180

${ }^{26}$ Vander Sloten J, Degryse K, Gobin R et al. Interactive simulation of cranial surgery in a computer aided design environment. J Craniomaxillofac Surg 1996; 24: 122-129

${ }^{27}$ Xia IJ, Shevchenko L, Gateno J et al. Outcome study of computer-aided surgical simulation in the treatment of patients with craniomaxillofacial deformities. J Oral Maxillofac Surg 2011; 69: 2014-2024

28 Zizelmann C, Hammer B, Gellrich NC et al. An evaluation of face-bow transfer for the planning of orthognathic surgery. J Oral Maxillofac Surg 2012; 70: 1944-1950

PD Dr. Dr. Majeed Rana

OA der MKG, stlv. Leiter der rekonstruktiven Gesichtschirurgie

Dipl.-Ing. Benjamin Singh

Forschungs- und Entwicklungsingenieur

Prof. Dr. Dr. Nils-Claudius Gellrich

Klinikdirektor

Klinik und Poliklinik für Mund-, Kiefer- und Gesichtschirurgie Medizinische Hochschule Hannover Carl-Neuberg-Straße 1 30625 Hannover

Rana.Majeed@mh-hannover.de 